\title{
Conical fireballs, cannonballs, and jet breaks in the afterglows of gamma ray bursts
}

\author{
Shlomo Dado and Arnon Dar \\ Physics Department, Technion, 32000 Haifa, Israel \\ Received 10 May 2013 / Accepted 17 August 2013

\begin{abstract}
The jet break in the X-ray afterglow of gamma ray bursts (GRBs) appears to be correlated to other properties of the X-ray afterglow and the prompt gamma ray emission, but the correlations are at odds with those predicted by the conical fireball (FB) model of GRBs. They are in good agreement, however, with those predicted by the cannonball (CB) model of GRBs.
\end{abstract}

Key words. gamma-ray burst: general

\section{Introduction}

Before the launch of the Compton Gamma Ray Observatory (CGRO) in 1991 it was widely believed that gamma-ray bursts (GRBs) originate in the Galaxy or in its halo. Much larger distances together with the observed fast rise time of GRB pulses implied an energy crisis - implausible energy release in gamma rays from a very small volume in a short time, if the emission was isotropic, as was generally assumed. However, the isotropic distribution of GRBs over the sky and their intensity distribution that were measured with the Burst and Transient Source Experiment (BATSE) aboard CGRO shortly after its launch provided clear evidence that the observed GRBs are at very large cosmological distances (Meegan et al. 1992; Mao \& Paczynski 1992). That led Shaviv \& Dar (1995) to propose that GRBs are produced by inverse Compton scattering of light by highly relativistic jets whose radiation is narrowly beamed along their direction of motion and that such jets are presumably ejected following violent stellar processes such as core collapse supernovae, merger of compact stars, mass accretion on compact stars and phase transition in compact stars, rather than in spherical fireballs (FBs; Paczynski 1986; Goodman 1986) produced by neutron star merger in close binaries (Goodman et al. 1987). These jets were assumed to be a succession of highly relativistic plasmoids of ordinary matter like those observed in high-resolution observations of highly relativistic jets ejected from galaxies with active galactic nucleus (e.g., M87 in Virgo), from radio galaxies (e.g., Centaurus A, the nearest radio galaxy), from quasars (e.g., 3C 273 in Virgo), and from microquasars (e.g., GRS 1915+105, SS 433, Cygnus X-1, and Cygnus $\mathrm{X}-3$ in our Galaxy). A key prediction of such a model was a very high linear polarization $(P \sim 100 \%)$ of gamma rays observed from the most probable viewing angle of the GRBs (Shaviv \& Dar 1995).

The hypothesis that GRBs are produced by highly relativistic jets was not widely accepted even when the discovery of the X-ray afterglows of GRBs by the Beppo-SAX satellite (Costa et al. 1997) allowed their arcminute localization, which led to the discovery of their longer wavelength afterglows (van Paradijs et al. 1997; Frail et al. 1997), host galaxies (Sahu et al. 1997), and their high redshifts (Metzger et al. 1997). In fact, the discovery of GRB afterglows, which appeared to decay like a single powerlaw in time, as predicted by Paczynski \& Rhoads (1993), Katz (1994) and Mezaros \& Rees (1997) from the isotropic FB model (Paczynski 1986; Goodman 1986), led to an immediate, wide acceptance of the relativistic isotropic FB model as the correct description of GRBs and their afterglows (see, e.g., Wijers et al. 1997; Piran 1999), ignoring the energy crisis of the isotropic FB model of GRBs.

When data on redshifts and afterglows of GRBs began to accumulate, it became clear that GRBs could not be explained by the isotropic FB model (e.g., Dar 1998). Not only did their high redshifts imply implausible energy release in gamma rays if the emission was isotropic, but their observed afterglows also seemed to behave like a smoothly broken powerlaw in time (Beuermann et al. 1999; Fruchter et al. 1999; Harrison et al. 1999; Kulkarni et al. 1999) rather than like a single power law. Only then was the isotropic FB replaced (e.g., Sari et al. 1999; Piran 1999, 2000) by an assumed conical jet of thin shells where synchrotron radiation from collisions between overtaking shells (or internal shocks) produce the observed GRB pulses, and the following collision of the merged shells with the interstellar medium (ISM) produces the synchrotron afterglow. This conical jet model which was given the name collimated FB model, replaced the original FB model, but retained its name the FB model.

The afterglow of a conical shell of opening angle $\theta_{j}$, whose propagation is decelerated by sweeping up the ISM in front of it, was shown by Sari et al. (1999) to have an achromatic break when its bulk motion Lorentz factor $\gamma(t)$ dropped below the value $\gamma(t)=1 / \theta_{j}$, which was argued to be roughly at the transition of the jet from a cone-like shape to a trumpet-like shape owing to the lateral expansion of the conical jet. Moreover, the conical FB model has been used to predict the pre- and postbreak temporal and spectral indices of the spectral energy density $F_{v}(t) \propto t^{-\alpha} v^{-\beta}$ of the afterglow and the closure relations that they satisfy.

Because of the complexity of the dynamics of spreading jets and the dependence of their afterglow on many adjustable parameters, the observed afterglows of GRBs rarely have been modeled with theoretical lightcurves calculated from the conical 
FB model. In most cases they were fitted with heuristic, sharply or smoothly broken power law functions connecting the pre- and post-break behaviors predicted by Sari et al. (1999). Such heuristic functions were used primarily for convenient parametrization of the data. They allowed, however, a break time $t_{\mathrm{b}}$ to be extracted from the observed lightcurve and to test whether the preand post-break slopes satisfy the closure relations of the conical FB model.

The jet breaks, however, were found to be chromatic (e.g., Covino et al. 2006; Panaitescu et al. 2006). The X-ray afterglows of GRBs with high equivalent isotropic energy $\left(E_{\text {iso }} \gg 10^{53} \mathrm{erg}\right)$ that were observed with the Swift X-ray telescope (XRT; e.g., GRBs 061007, 130427A) showed a single power law behavior with no visible jet break, and almost all the X-ray afterglows of less energetic GRBs that appeared to have a jet break did not satisfy the closure relations of the conical FB model, either before the break or after it (e.g., Liang et al. 2008; Racusin et al. 2009). In particular, a large portion of the X-ray afterglows of GRBs measured with the Swift X-ray telescope (Swift/XRT) showed canonical behavior (Nousex et al. 2006) where the afterglow has a shallow decay phase (plateau) before the break with $\alpha\left(t<t_{\mathrm{b}}\right) \ll 1$ far from the predicted $\alpha_{\mathrm{X}}\left(t<t_{\mathrm{b}}\right)=\left(3 \beta_{\mathrm{X}}-1\right) / 2$. Despite these and many other failures of the FB model, the model has not been given up. Instead, the missing breaks were attributed to various reasons, such as quality of the data (Curran et al. 2008), break time beyond the end of the Swift/XRT followup observations (Kocevski \& Butler 2008), and far off-axis observations (Van Eerten et al. 2011). The failure of the pre-break closure relation to describe the shallow decay/plateau phase of canonical X-ray afterglows was attributed to an assumed continuous energy injection. The chromaticity of the jet break and the failure of the closure relation for the post-break behavior of the $\mathrm{X}$-ray afterglow were largely ignored.

To test whether part of the above difficulties arise from approximations used in the analytical calculations and to generalize the predictions to off-axis observers, various authors have tried to derive the lightcurves of conical FBs from numerical hydrodynamical calculations. In particular, van Eerten \& MacFadyen (2013) have recently reported two dimensional (2D) numerical hydrodynamic calculations of the lightcurves of the afterglow from conical FBs observed from an arbitrary angle. These numerical calculations showed that the difference in the temporal indices across the jet break is greater than predicted by Sari et al. (1999) and, contrary to expectations, it increases the discrepancy between theory and observations rather than removing it. The pre-break behavior remains an unsolved difficulty, which was speculated to be due to an assumed continuous energy injection into the conical FB. It was also speculated that the discrepancy between the post-break temporal slopes obtained from the numerical simulations and those observed with the Swift/XRT may be removed or reduced by assuming that the afterglow is produced by a blast wave that decelerates in a wind environment rather than in a constant density ISM.

All the above difficulties of the conical FB model, however, were not shared by the cannonball (CB) model of GRBs: The canonical behavior of X-ray afterglows where a plateau/shallow decay phase is smoothly broken to a steep power law decline was predicted long before it was observed with Swift/XRT (see, e.g., Figs. 6, 26-30 in Dado et al. 2002; see also Dado et al. 2009 a,b for a detailed comparison between the lightcurves of the X-ray afterglows of GRBs measured with Swift/XRT and those predicted by the CB model). The post-break closure relations predicted by the $\mathrm{CB}$ model were also shown to be satisfied well by the Swift/XRT light curves (Dado \& Dar 2012a).
The failures of the standard conical FB model, however, did not appear to shake the wide belief in this model or in its interpretation of the afterglow breaks. In this paper we present therefore additional parameter-free tests of the origin of the observed break in the lightcurve of canonical X-ray afterglows of GRBs, by comparing the observed correlations between the jet breaks in the X-ray afterglows of GRBs measured with the Swift/XRT between December 2004 and December 2012 and the prompt gamma-ray emission properties of these GRBs, and those predicted by the conical FB model and the CB model. We limit our tests to the X-ray afterglow, in order to avoid dependence on adjustable parameters. This extends our preliminary study of missing breaks in the X-ray afterglows of GRBs (Dado et al. 2007) that was based on limited statistics. For completeness, the derivation of the break properties from the conical FB model and from the $\mathrm{CB}$ model are presented in Appendices A1 and A2, respectively.

\section{Jet break correlations}

\subsection{Conical jet break}

In standard conical jet models of GRBs, $1 / \gamma(0) \ll \theta_{j}$. The break in the afterglow occurs when the beaming angle $1 / \gamma(t)$ of the emitted radiation from the decelerating jet in the ISM becomes larger than the opening angle $\theta_{j}$ of the conical jet, i.e., when $1 / \gamma\left(t_{\mathrm{b}}\right) \approx \theta_{j}$. For a conical jet at redshift $z$ with kinetic energy $E_{\mathrm{k}}$ propagating in an ISM with a constant baryon density $n_{\mathrm{b}}$, the break is observed by a distant observer on or near the axis at a time (see Appendix A)

$t_{\mathrm{b}} \approx \frac{(1+z)}{8 c}\left[\frac{3 E_{\mathrm{k}}}{2 \pi n_{\mathrm{b}} m_{\mathrm{p}} c^{2}}\right]^{1 / 3} \theta_{j}^{2}$.

Equation (1) is the relation derived by Sari et al. (1999) for a conical shell that begins rapid lateral spreading on top of its radial motion when $\gamma(t) \approx 1 / \theta_{j}$.

If the jets that produce GRBs approximately had a standard ISM environment and a standard $E_{\mathrm{k}}$ (Frail et al. 2001), then Eq.(1) would have yielded the correlation

$t_{\mathrm{b}}^{\prime} \propto\left[E_{\mathrm{iso}}\right]^{-1}$,

in the GRB rest frame where $E_{\text {iso }}$ is the total gamma-ray energy emission under the assumption of isotropic emission.

Because $1 / \gamma(0) \ll \theta_{j}$, the highly relativistic conical ejecta and its beamed gamma-ray emission share the same cone. Since the observed spectrum of GRBs is given roughly by a cutoff powerlaw (CPL) $E \mathrm{~d} n_{\gamma} / \mathrm{d} E \propto \mathrm{e}^{-E / E_{\mathrm{p}}}$, the assumption of the conical FB model that a constant fraction of the jet kinetic energy is converted to gamma-ray energy implies that

$E_{\mathrm{p}}^{\prime} \propto \frac{E_{\mathrm{k}}}{\pi \theta_{j}^{2}} \propto E_{\mathrm{iso}}$

where $E_{\mathrm{p}}^{\prime}$ is the peak energy of the time-integrated observed spectral energy flux. Consequently, the FB model assumptions also yield the binary correlation $t_{\mathrm{b}}^{\prime} \propto\left[E_{\mathrm{p}}^{\prime}\right]^{-1}$.

Also for a ballistic (non spreading) conical jet viewed as above, the power law decline of the X-ray afterglow of an isotropic FB seen by a distant observer is multiplied by a factor

$K_{\mathrm{b}}=\left[1-\frac{1}{\left(1+\gamma^{2} \theta_{j}^{2}\right)^{\beta_{\mathrm{X}}+1}}\right]$, 
which follows from Eq. (A.6) of Appendix A1 for $\theta_{j}^{2} \ll 1$. For $t \ll t_{\mathrm{b}}, \gamma^{2} \theta_{j}^{2} \gg 1$ and $K_{\mathrm{b}} \approx 1$, while for $t \gg t_{\mathrm{b}}, \gamma^{2} \theta_{j}^{2} \ll 1$ and $K_{\mathrm{b}} \approx\left(\beta_{\mathrm{X}}+1\right) \gamma^{2} \theta_{j}^{2} \propto t^{-3 / 4}$. The temporal index $\alpha$ of the afterglow of a conical jet thus increases by $\Delta \alpha=0.75$ across the break, independent of the spectral index $\beta_{\mathrm{X}}$ and the pre-break temporal index of the afterglow.

In the case of a wind-structured environment with a density profile $n(R)=n_{0} R_{0}^{2} / R^{2}$, the power law indices of the $t_{\mathrm{b}}^{\prime}-E_{\text {iso }}$ and $t_{\mathrm{b}}^{\prime}-E_{\mathrm{p}}^{\prime}$ correlations are identical to those for an ISM circumburst environment (see Appendix A). Moreover, although the $t_{\mathrm{b}}^{\prime}-E_{\text {iso }}$ and $t_{\mathrm{b}}^{\prime}-E_{\mathrm{p}}^{\prime}$ correlations were derived for the case where no continuous energy injection during the plateau phase of the X-ray afterglow takes place, they are valid also, to a good approximation, when continuous energy injection is invoked. This is because the injected energy during the afterglow phase must be much smaller than the initial kinetic energy that powers the prompt gamma-ray emission whose energy is is much larger than that of the afterglow. Since the afterglow is only partially powered by the assumed continuous energy injection, the continuous energy injection must be rather small compared to $E_{\mathrm{k}}$, the total kinetic energy of the jet. The assumption, $E_{\mathrm{iso}} \propto E_{\mathrm{k}}$ therefore holds to a good approximation and Eq. (A.1) implies that the correlations derived from it are also valid when such a continuous energy injection after the prompt emission phase is present.

\subsection{Cannonball deceleration break}

In the CB model of GRBs, a succession of initially expanding plasmoids (CBs) of ordinary matter merge into a slowly expanding $\left(\left(k T / m_{\mathrm{p}} \gamma^{2}\right)^{1 / 2} \ll c\right)$ leading $\mathrm{CB}$ with a large bulk motion Lorentz factor, $\gamma(0) \sim 10^{3}$, that decelerates in collision with the circumburst medium/ISM. The emitted synchrotron radiation is relativistically beamed along its direction of motion, redshifted by the cosmic expansion and its arrival time in the frame of a distant observer at a viewing angle $\theta$ relative to the direction of motion of the CB is aberrated (e.g., Dar \& De Rújula 2004, and references therein). The rate of change in the bulk motion Lorentz and Doppler factors of the $\mathrm{CB}$ due to the deceleration of the $\mathrm{CB}$ in the circumburst medium is low until the swept-in mass by the $\mathrm{CB}$ becomes comparable to its initial mass. This happens in the observer frame at a time (see, e.g., Dado et al. 2009a, and references therein)

$t_{\mathrm{b}} \approx \frac{(1+z) N_{\mathrm{b}}}{8 c n_{\mathrm{b}} \pi R^{2}} \frac{1}{\gamma_{0} \delta_{0}^{2}}$,

where $N_{b}$ is the baryon number of the $\mathrm{CB}$ and $R$ its radius. The rapid decrease of $\gamma(t)$ and $\delta(t)$ beyond $t_{\mathrm{b}}$ produces a smooth transition (break) around $t_{\mathrm{b}}$ of the observed spectral energy density $F_{v}(t) \propto[\gamma(t)]^{3 \beta-1}[\delta(t)]^{\beta+3} v^{-\beta}$ of the emitted afterglow from a plateau phase to an asymptotic power law decline (e.g., Dado et al. 2002, 2009a, and references therein).

In the $\mathrm{CB}$ model, $E_{\mathrm{p}}^{\prime} \propto \gamma_{0} \delta_{0}$ and $E_{\mathrm{iso}} \propto \gamma_{0} \delta_{0}^{3}$, respectively. Consequently, Eq. (5) yields the triple correlation,

$t_{\mathrm{b}}^{\prime} \propto\left[E_{\mathrm{p}}^{\prime} E_{\mathrm{iso}}\right]^{-1 / 2}$.

Moreover, substituting the $\mathrm{CB}$ model's approximate binary power law correlation $E_{\mathrm{p}}^{\prime} \propto\left[E_{\mathrm{iso}}\right]^{1 / 2}$ into the triple $t_{\mathrm{b}}^{\prime}-E_{\mathrm{p}}^{\prime}-E_{\mathrm{iso}}$ correlation yields the $t_{\mathrm{b}}^{\prime}-E_{\mathrm{iso}}$ and $t_{\mathrm{b}}^{\prime}-E_{\mathrm{p}}^{\prime}$ binary correlations,

$t_{\mathrm{b}}^{\prime} \propto\left[E_{\mathrm{iso}}\right]^{-3 / 4} \propto\left[E_{\mathrm{p}}^{\prime}\right]^{-3 / 2}$

where a prime indicates the value in the GRB rest frame. Naturally, these approximate correlations are expected to have a wider spread than the original triple $t_{\mathrm{b}}^{\prime}-E_{\mathrm{p}}^{\prime}-E_{\text {iso }}$ correlation.

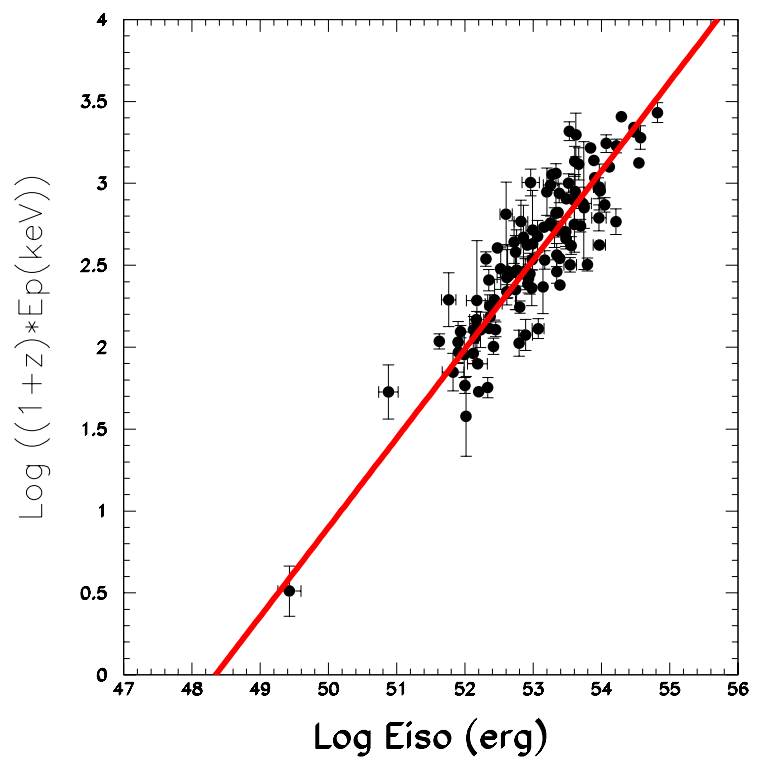

Fig. 1. Observed correlation between $E_{\mathrm{p}}^{\prime}$ and $E_{\text {iso }}$ for 121 long GRBs with known redshift. The best fit power law correlation (straight line) has a power law index 0.54 .

In the case of a wind-like density, which typically extends beyond the end of the glory region at $R_{\mathrm{g}} \sim 10^{16} \mathrm{~cm}$ (Dado et al. $2009 \mathrm{a}, \mathrm{b})$ and up to $R_{w} \sim 5 \times 10^{17} \mathrm{~cm}$ where the wind density decreases below $\sim m_{\mathrm{p}} / \mathrm{cm}^{3}$ (for the wind parameters listed in Appendix A), the predicted spectral energy flux of the afterglow has the behavior $F_{v}(t) \propto t^{-(\beta+1)} v^{-\beta}$ where $\beta(t)$ is the spectral index in the observed band (see, e.g., Dado et al. 2009a,b). The observed crossing time of such a wind region is roughly $(1+z) R_{w} / \gamma_{0} \delta_{0}$, typically $<50(1+z)$ s because $\gamma$ and $\delta$ change little during the wind crossing. Beyond the wind region, $F_{v}(t)$ in the X-ray band has the standard canonical behavior of X-ray afterglows in the CB model in an ISM environment, i.e., with an afterglow smooth break/bend at the end of a plateau phase that satisfies Eq. (7).

\section{Comparison with observations}

Figure 1 presents the best fit power law to the observed $E_{\mathrm{p}}^{\prime}-E_{\text {iso }}$ correlation using an unbiased sample of 110 GRBs with known redshift measured before January 1,2013 . The values of $E_{\mathrm{p}}^{\prime}$ and $E_{\text {iso }}$ were compiled from communications of the KonusWind and Fermi GBM collaborations to the GCN Circulars Archive (Barthelmy 1997), and from publications by Amati et al. (2007, 2008), Yonetoku et al. (2010), Gruber et al. (2011), Nava et al. (2012), and D'Avanzo et al. (2012). Using essentially the method advocated by D'Agostini (2005), we obtained the best fit power law correlations $E_{\mathrm{p}}^{\prime} \propto\left[E_{\mathrm{iso}}\right]^{0.54}$, in good agreement with $E_{\mathrm{p}}^{\prime} \propto\left[E_{\mathrm{iso}}\right]^{1 / 2}$ predicted by the $\mathrm{CB}$ model but in disagreement with $E_{\mathrm{p}}^{\prime} \propto E_{\text {iso }}$ expected in the conical FB model.

Figure 2 compares the triple correlation $t_{\mathrm{b}}^{\prime}-E_{\mathrm{p}}^{\prime}-E_{\text {iso }}$ predicted by the CB model (Eq. (6)) and the observed correlation in 70 Swift GRBs (Evans et al. 2009) from the above GRB sample, which have a good Swift/XRT temporal sampling of their $\mathrm{X}$-ray afterglow during the first day at least following the prompt emission phase and have no superimposed flares. In this sample the X-ray afterglow of 55 GRBs clearly show a break and no afterglow-break was observed in 15 GRBs. The upper bound on a possible early time break for the $15 \mathrm{GRBs}$ with no visible break are indicated. Also shown is the late-time break of the X-ray 


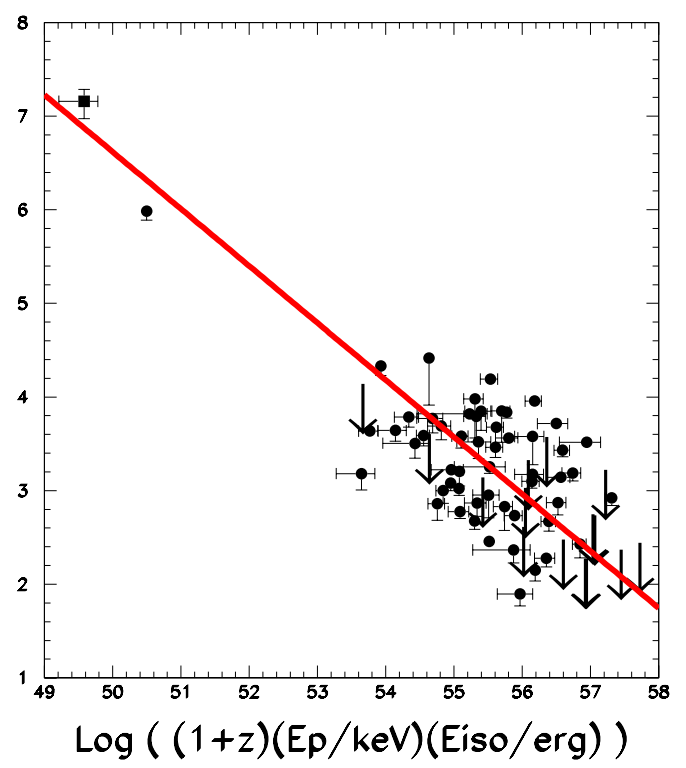

Fig. 2. Observed triple correlations $t_{\mathrm{b}}^{\prime}-E_{\mathrm{p}}^{\prime}-E_{\text {iso }}$ in 70 Swift GRBs with measured redshift, $t_{\mathrm{b}}^{\prime}, E_{\mathrm{p}}^{\prime}$, and $E_{\text {iso }}$ and its best fit power law (straight line with a power law index -0.58$)$. Arrows indicate observational upper bounds on early time deceleration breaks before the beginning of the Swift/XRT observations or hidden under the prompt emission tail. The square represents the break in the late-time X-ray afterglow of GRB 980425 which was observed with Chandra.

afterglow of GRB 980425, which was measured with Chandra (Kouveliotou et al. 2004). In order not to bias the values of $t_{\mathrm{b}}$ by the CB model fits, the break times were taken to be the times of the first break with $\alpha\left(t<t_{\mathrm{b}}\right)<\alpha\left(t>t_{\mathrm{b}}\right)$ obtained from a broken power law fit to the GRB X-ray afterglow measured with the Swift/XRT and reported in the Leicester XRT GRB catalog (Evans et al. 2009) or from the smoothly broken power law fits of Margutti et al. (2013). The Spearman rank (correlation coefficient) of the triple correlation $t_{\mathrm{b}}^{\prime}-\left(E_{\mathrm{p}}^{\prime} E_{\mathrm{iso}}\right)$ for the subsample of 55 GRBs with a visible break is $r=-0.74$ corresponding to a chance probability less than $1.4 \times 10^{-10}$. The best fit triple correlation $t_{\mathrm{b}}^{\prime} \propto\left[E_{\mathrm{p}}^{\prime} E_{\mathrm{iso}}\right]^{q}$ that was obtained for the subsample of 55 GRBs, using essentially the maximum likelihood method advocated by D'Agostini (2005), yields $q=-0.58 \pm 0.04$.

The approximate binary correlations $t_{\mathrm{b}}^{\prime}-E_{\mathrm{iso}}$ and $t_{\mathrm{b}}^{\prime}-E_{\mathrm{p}}^{\prime}$ that were obtained by substituting the $\mathrm{CB}$ model predicted correlation $E_{\mathrm{p}}^{\prime} \propto\left[E_{\mathrm{iso}}\right]^{1 / 2}$ in the triple correlation $t_{\mathrm{b}}^{\prime}-E_{\mathrm{p}}^{\prime}-E_{\text {iso }}$ (Eq. (6)) are compared to the observational data in Figs. 3 and 4 , respectively. GRB 980425 was excluded from the GRB sample because in the $\mathrm{CB}$ model $E_{\mathrm{p}}^{\prime} \propto\left[E_{\mathrm{iso}}\right]^{1 / 2}$ is only satisfied by ordinary GRBs where $\theta \approx 1 / \gamma$, while far-off axis GRBs, such as 980425 , with $\theta \gg 1 / \gamma$, satisfy $E_{\mathrm{p}}^{\prime} \propto\left[E_{\mathrm{iso}}\right]^{1 / 3}$; i.e., they are outliers with respect to the assumed $E_{\mathrm{p}}^{\prime} \propto\left[E_{\mathrm{iso}}\right]^{1 / 2}$ correlation. The Spearman ranks of the observed $t_{\mathrm{b}}^{\prime}-E_{\mathrm{iso}}$ and $t_{\mathrm{b}}^{\prime}-E_{\mathrm{p}}^{\prime}$ correlations are -0.49 and -0.63 with chance probabilities less than $4.5 \times 10^{-4}$ and $1.0 \times 10^{-6}$, respectively, and as expected (in the CB model), they are larger than that of the $t_{\mathrm{b}}^{\prime}-\left(E_{\mathrm{p}}^{\prime} E_{\mathrm{iso}}\right)$ correlations. The best fit power law indices of the $t_{\mathrm{b}}^{\prime}-E_{\mathrm{iso}}$, and $t_{\mathrm{b}}^{\prime}-E_{\mathrm{p}}^{\prime}$ correlations are $-0.70 \pm 0.06$, and $-1.64 \pm 0.04$, respectively, consistent with their predicted values by the CB model, -0.75 and -1.50 , respectively.

A comparison between the best fit indices of the breaktime power law correlations and those expected in the $\mathrm{CB}$ and FB models is summarized in Table 1 for the sample of 55 Swift GRBs with a visible afterglow break. As can be seen from

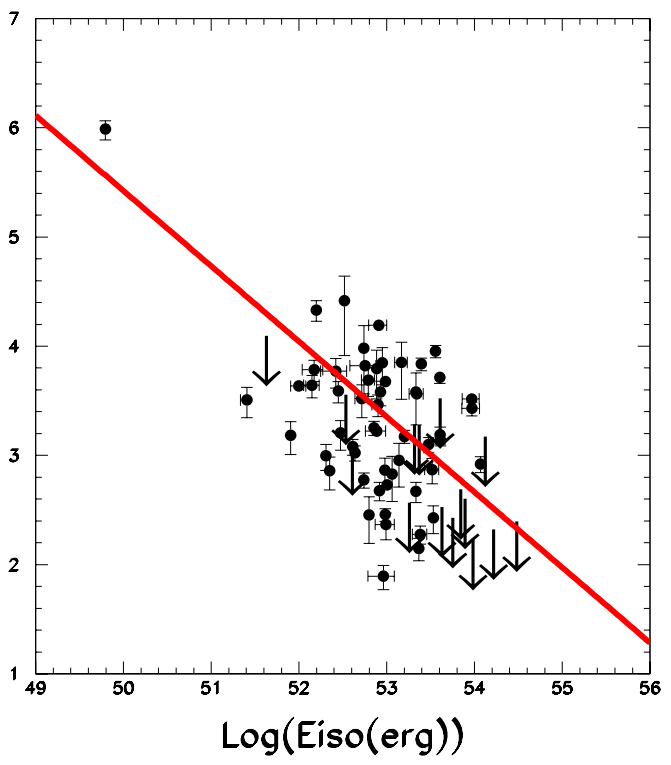

Fig. 3. Comparison between the binary correlation $t_{\mathrm{b}}^{\prime}-E_{\text {iso }}$ predicted by the CB model (Eq. (7)) and that observed in 70 Swift GRBs with known redshift, $t_{\mathrm{b}}^{\prime}$ and $E_{\mathrm{iso}}$. Arrows indicate observational upper bounds on early time deceleration breaks before the beginning of the Swift/XRT observations or hidden under the prompt emission tail.

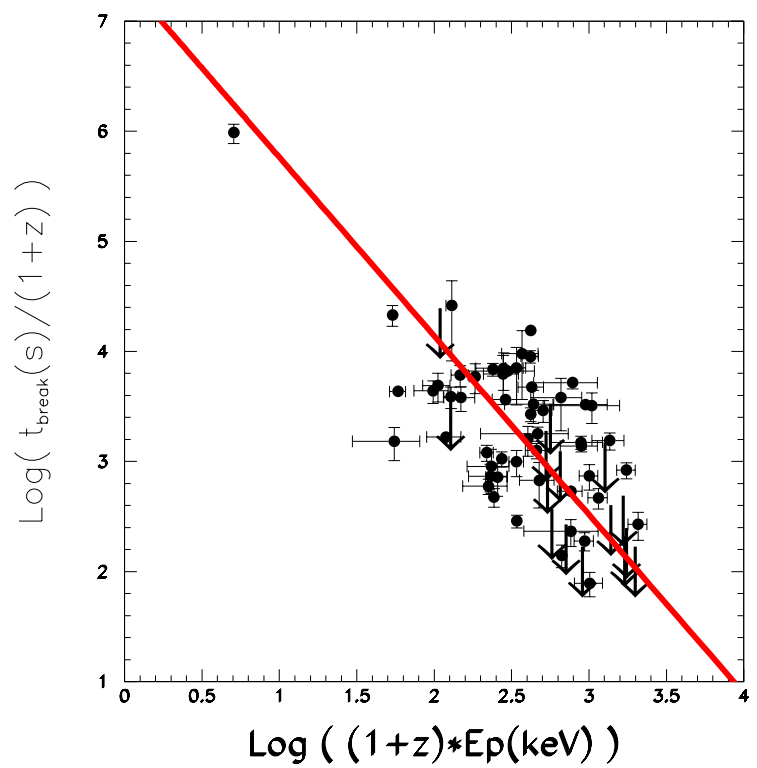

Fig. 4. Comparison between the binary correlation $t_{\mathrm{b}}^{\prime}-E_{\mathrm{p}}^{\prime}$ predicted by the CB model (Eq. (7)) and that observed in 70 Swift GRBs with known redshift, $t_{\mathrm{b}}^{\prime}$ and $E_{\mathrm{p}}^{\prime}$. Arrows indicate observational upper bounds on early time deceleration breaks that may have taken place before the beginning of the Swift/XRT observations or are hidden under the prompt emission tail.

Table 1, the values of the power law correlation indices predicted by the CB model are consistent with those obtained from the best fits. The best fit indices, $0.54 \pm 0.01,-0.69 \pm 0.06$, and $-1.62 \pm 0.04$ of the observed $E_{\mathrm{p}}^{\prime}-E_{\mathrm{iso}}, t_{\mathrm{b}}^{\prime}-E_{\mathrm{p}}^{\prime}$ and $t_{\mathrm{b}}^{\prime}-E_{\text {iso }}$ power law correlations, however, are at odds with the values $1,-1$, and -1 , respectively, expected in the conical FB model. While the $\chi^{2} /$ d.o.f. of the predicted correlations by the CB model differ from those of the best fits by less than $1 /$ d.o.f., the $\chi^{2} /$ d.o.f. of the predicted correlations by the FB model differ by much higher values, as summarized in Table 2. 
Table 1. Summary of the observed power law correlations between $E_{\mathrm{p}}^{\prime}$, $E_{\text {iso }}$ and $t_{\mathrm{b}}^{\prime}$ and their power law indices expected in the cannonball (CB) and collimated fireball (FB) models.

\begin{tabular}{lccccc}
\hline \hline Correlation & $\rho$ & $P(\rho)$ & $p($ fit $)$ & $p(\mathrm{CB})$ & $p(\mathrm{FB})$ \\
\hline$E_{\mathrm{p}}^{\prime}-E_{\text {iso }}$ & +0.87 & $\sim 0$ & $0.54 \pm .01$ & $1 / 2$ & 1 \\
$t_{\mathrm{b}}^{\prime}-\left(E_{\mathrm{p}}^{\prime} E_{\text {iso }}\right)$ & -0.74 & $1.3 \times 10^{-10}$ & $-0.58 \pm .04$ & $-1 / 2$ & \\
$t_{\mathrm{b}}^{\prime}-E_{\mathrm{p}}^{\prime}$ & -0.63 & $1.0 \times 10^{-6}$ & $-1.62 \pm .04$ & $-3 / 2$ & -1 \\
$t_{\mathrm{b}}^{\prime}-E_{\text {iso }}$ & -0.49 & $4.5 \times 10^{-4}$ & $-0.69 \pm .06$ & $-3 / 4$ & -1 \\
\hline
\end{tabular}

Notes. $\rho$ is the Spearman rank (corrrelation coefficient), $P(\rho)$ is the chance probability of a correlation coefficient $\geq \rho$, and $p$ is the power law index of the power law correlation.

Table 2. The $\chi^{2} /$ d.o.f. statistic for the best fit power law correlations between $E_{\mathrm{p}}^{\prime}, E_{\mathrm{iso}}$ and $t_{\mathrm{b}}^{\prime}$ and for the correlations predicted by the $\mathrm{CB}$ and collimated FB models.

\begin{tabular}{lcccc}
\hline \hline Correlation & d.o.f. & Best fit & CB & FB \\
\hline$E_{\mathrm{p}}^{\prime}-E_{\text {iso }}$ & 108 & 19.2 & 20.2 & 99.7 \\
$t_{\mathrm{b}}^{\prime}-\left(E_{\mathrm{p}}^{\prime} E_{\text {iso }}\right)$ & 53 & 26.9 & 27.5 & - \\
$t_{\mathrm{b}}^{\prime}-E_{\mathrm{p}}^{\prime}$ & 53 & 22.3 & 23.1 & 27.6 \\
$t_{\mathrm{b}}^{\prime}-E_{\text {iso }}$ & 53 & 23.0 & 24.6 & 30.1 \\
\hline
\end{tabular}

The correlations satisfied by $t_{\mathrm{b}}^{\prime}$ imply that GRBs with very high $E_{\text {iso }}$ and/or $E_{\mathrm{p}}^{\prime}$ have a break at small $t_{\mathrm{b}}^{\prime}$, which is hidden under the tail of the prompt emission or precedes the start of the Swift/XRT follow-up observations (Dado et al. 2007). Indeed, the X-ray afterglow of all the 15 GRBs in the sample, which have a very high $E_{\text {iso }} \times E_{\mathrm{p}}^{\prime}$, have a single power law decline consistent with the post-break power law decline predicted by the CB model (see, e.g., Dado et al. 2007, 2009a). For such GRBs, the observations only provide upper bounds for the break times of their X-ray afterglows.

However, for most of the 15 GRBs with only an upper bound on their afterglow break time, which are indicated by down pointing errors in Figs. 2-4, an early break time value was extracted from a CB model fit to the entire X-ray light curve, which includes the fast decline phase of the prompt emission and the afterglow component (see, e.g., Dado et al. 2009a,b). Replacement of the upper bounds by the CB model fitted break times (which could have biased the break times values) and their inclusion in the best fits had very little effect on the values of the best fit power law indices and their errors.

\section{Conclusions and discussion}

Correlations and closure relations between GRB properties that are predicted by GRB models allow parameter-free tests of such models. In particular, comparison between the observed and predicted correlations between the jet break in the X-ray afterglow of GRBs and the prompt gamma-ray emission, like the $E_{\mathrm{p}}^{\prime}-E_{\text {iso }}$ correlation, allow another critical test of the conical FB model and the CB model of GRBs. Although the jet break in the afterglow of GRBs has been the flagship of the conical FB model, the observed correlations between the jet break in the X-ray afterglow of GRBs measured with the Swift/XRT and their prompt gamma-ray emission are inconsistent with those expected in the conical FB model. This failure, perhaps, is not a surprise since the observed jet-breaks were found before to be chromatic, the predicted pre- and post-break temporal behaviors and closure relations were found to be badly violated, and the observed change in slope across the breaks is not the one predicted.
The replacement of the approximate analytical estimates in the conical FB model (Sari et al. 1999) by more exact hydrodynamical calculations (e.g. van Eerten \& MacFadyen 2013) does not change the situation. They neither reproduce the observed correlations, nor do they remove the discrepancies between the predicted and observed pre-break and post break behaviors of the afterglows. These failures provide additional evidence that GRBs and their afterglows are not produced by conical jets, the so called collimated FBs.

In contrast, the correlations between the deceleration break in the afterglow of GRBs and their prompt $\gamma$-ray emission predicted by the CB model are in good agreement with those observed, as shown in Figs. 1-4. The correlations between the break and other afterglow properties predicted by the CB model (Dado \& Dar 2012b), as well as the pre-break and post-break behaviors of the light curves of the X-ray afterglow, were shown to accord well with the observations (e.g., Dado et al. 2009a,b; Dado \& Dar 2012a). Moreover, in the CB model, the $t_{\mathrm{b}}^{\prime}-(1+z)$ anti-correlation noted by Stratta et al. (2009), is a simple consequence of beaming and the detection threshold, which enrich the low $z$ GRB sample with far-off-axis soft GRBs and X-ray flashes (e.g., Dado et al. 2004) relative to the high $z$ events that must be much harder and energetic in order to be detected. These selection effects that produce the effective $\left\langle E_{\mathrm{p}}^{\prime}(z)\right\rangle-z$ and $\left\langle E_{\text {iso }}(z)\right\rangle-z$ "correlations" result in an effective $t_{\mathrm{b}}^{\prime}(z)-z$ 'anti-correlation' (Dado \& Dar, in prep.).

Acknowledgements. We thank an anonymous referee for useful comments and suggestions.

\section{Appendix A: Ballistic conical shells}

Consider the deceleration of a highly relativistic conical shell of a solid angle $2 \pi\left(1-\cos \theta_{j}\right) \approx \pi \theta_{j}^{2}$ that expands radially and decelerates by sweeping in the medium in front of it. Assuming a plastic collision and neglecting radiation losses, relativistic energy-momentum conservation, $\mathrm{d}(M \gamma)=0$, can be written as,

$\frac{M_{0} \gamma_{0}}{\gamma} \mathrm{d} \gamma=-\gamma^{2} \pi \theta_{j}^{2} n_{\mathrm{b}} m_{\mathrm{p}} R^{2} \mathrm{~d} R$

where $M(t)=M_{0} \gamma(0) / \gamma$ is the mass of the jet, $M_{0}=M(0), \gamma_{0}=$ $\gamma(0), n_{\mathrm{b}}$ is the constant baryon density of the external medium, and $R(t)$ is the radius of the conical shell. Equation (A.1) yields

$\left[R^{3}-R_{0}^{3}\right]=\frac{3 M_{0} \gamma_{0}}{2 \pi \theta_{j}^{2} n m_{\mathrm{p}}}\left[\frac{1}{\gamma^{2}}-\frac{1}{\gamma_{0}^{2}}\right]$.

In the conical FB model, ordinary GRBs have $\gamma_{0}^{2} \theta_{j}^{2} \gg 1$, whereas $\gamma\left(t_{\mathrm{b}}^{\prime}\right) \theta_{j} \approx 1$ at the break-time $t_{\mathrm{b}}^{\prime}$ in the GRB rest frame corresponding to a break-time $t_{\mathrm{b}}$ in the observer frame. Consequently, Eq. (A.1) implies that the radius of the conical shell at $t_{\mathrm{b}}^{\prime}$ is given by

$R_{\mathrm{b}}=R\left(t_{\mathrm{b}}^{\prime}\right) \approx\left[\frac{3 E_{\mathrm{k}}}{2 \pi n m_{\mathrm{p}} c^{2}}\right]^{1 / 3}$,

where $E_{\mathrm{k}} \simeq M_{0} \gamma_{0} c^{2}$ is the kinetic energy of the conical ejecta. Equation (A.1) also yields the asymptotic behavior $R \approx$ $c t^{\prime} \approx R_{\mathrm{b}}\left[\gamma\left(t^{\prime}\right) / \gamma_{\mathrm{b}}\right]^{-2 / 3}$, which is already reached well before $t_{\mathrm{b}}^{\prime}$. Because of time aberration and cosmic expansion, the on-axis observer's time interval $\mathrm{d} t$ that corresponds to the time interval $\mathrm{d} t^{\prime}=\mathrm{d} R / c$ in the GRB rest frame is given by $\mathrm{d} t=(1+z) \mathrm{d} t^{\prime} / 2 \gamma^{2}$. 
Consequently, $\gamma\left(t^{\prime}\right)$ has the asymptotic behavior $\gamma_{\mathrm{b}}\left(t / t_{\mathrm{b}}\right)^{-3 / 8}$ where the observed on-axis break-time is given by

$t_{\mathrm{b}}=\int^{t_{\mathrm{b}}} \mathrm{d} t=(1+z) \int^{t_{\mathrm{b}}^{\prime}} \frac{\mathrm{d} t^{\prime}}{2 \gamma^{2}} \approx \frac{(1+z) R_{\mathrm{b}} \theta_{j}^{2}}{8 c}$

and where we have assumed that most of the contribution to the integral comes from times when $\gamma\left(t^{\prime}\right)=\gamma_{\mathrm{b}}\left(t^{\prime} / t_{\mathrm{b}}^{\prime}\right)^{-3 / 2}$ is already a good approximation.

If the total gamma-ray energy emitted in GRBs is a constant fraction of the initial kinetic energy of the conical shell, $E_{\gamma}=\eta E_{\mathrm{k}}$, which in the conical FB model is related to the isotropic equivalent gamma-ray energy $E_{\text {iso }}$ of the GRB by ${ }^{1}$ $E_{\text {iso }} \approx 4 E_{\gamma} / \theta_{j}^{2}$ then

$t_{\mathrm{b}}=\frac{(1+z)}{16 c}\left[\frac{3 E_{\mathrm{iso}}}{\pi \eta n m_{\mathrm{p}} c^{2}}\right]^{1 / 3} \theta_{j}^{8 / 3}$.

Equations (A.4) and (A.5) were used by Sari et al. (1999) to represent conical jets with lateral expansion.

A distant observer sees only the beamed radiation from an area $R^{2} \pi / \gamma^{2}$ along the line of sight of a spherical shell or a conical shell. Consequently, as long as $\gamma(t)>1 / \theta_{j}$ the observed afterglows from a conical FB or an isotropic FB have the same visible area. Beyond the break the visible area of a spherical FB continues to be $\approx R^{2} \pi / \gamma^{2}$, while that of a conical shell becomes $\approx R^{2} \pi \theta_{j}^{2}$. Hence the lightcurve of the afterglow of the conical FB beyond the break is steeper by their ratio $\gamma^{2} \theta_{j}^{2} \approx\left(t / t_{\mathrm{b}}\right)^{-0.75}$, where we used the asymptotic behavior $\gamma(t)=\gamma_{\mathrm{b}}\left(t / t_{\mathrm{b}}\right)^{-3 / 8}$ in a constant density environment and $\gamma_{\mathrm{b}}=1 / \theta_{j}$. This steepening of the power law decline by $\Delta \alpha=0.75$ across the break independent of $\beta$ is different from what was derived by Sari et al. (1999) for a spreading jet.

The smooth transition between the pre- and post-break power laws can also be derived more rigorously: relativistic beaming and Doppler boosting modulates the observed emission from every point on the conical shell by a factor $\delta^{1+\Gamma}$ where $\delta=1 / \gamma(1-\beta \cos \theta)$ is the Doppler factor on the shell at an angle $\theta$ relative to the line of sight to the observer, $\beta=v / c$, and $\Gamma$ is the photon spectral index of the radiation. For isotropic medium, isotropic conical shell, and isotropic expansion, this is the only dependence of the received radiation on the line of sight to the observer. Consequently, the observed energy-flux (at a given energy) of photons emitted simultaneosly by the conical shell is modulated by the factor

$I\left(\gamma, \theta_{j}\right)=2 \pi \int \delta^{\Gamma+1} \mathrm{~d} \cos \theta=\frac{2 \pi}{\beta \gamma \Gamma}\left[\frac{1}{(1-\beta)^{\Gamma}}-\frac{1}{\left(1-\beta \cos \theta_{j}\right)^{\Gamma}}\right]$.

The difference in arrival times of photons emitted simultaneously from the conical shell was ignored in the above analytical estimates of the break time and the spectral index change across the break. The spread in arrival times has no effect on $E_{\text {iso }}$, it is $\Delta t \approx R / 2 c \gamma^{2}$ before the break and $\Delta t \approx R \theta_{j}^{2} / c$ after the break. Thus, $\Delta t$ is roughly four times higher than $t$ before the break but less than $t$ by a factor $4 \gamma^{2} \theta_{j}^{2} \ll 1$ at late times. The spread in arrival time that has a negligible effect on $E_{\text {iso }}$ and $\Delta \alpha$ cannot be neglected in estimating $t_{\mathrm{b}}$. The same conclusion is also valid

\footnotetext{
1 The solid angle of a conical shell is $\Omega_{j}=2 \pi\left(1-\cos \theta_{j}\right)$, which yields a beaming factor $f_{\mathrm{b}}=2 \pi\left(1-\cos \theta_{j}\right) / 4 \pi=\left(1-\cos \theta_{j}\right) / 2$, and not $f_{\mathrm{b}}=\left(1-\cos \theta_{j}\right)$ that is widely used in the GRB literature.
}

for the effects of off-axis viewing when the viewing angle $\theta$ is not negligible compared to $\theta_{j}$. Generally, the effects of off-axis viewing and the spread in arrival time require numerical integrations (e.g., van Eerten \& MacFadyen 2013) and make the widely used simple relation, Eq. (A.4) a very rough estimate.

If the typical circumburst region of LGRBs is the wind region of a Wolf Rayet star that blows a constant wind, then its density profile is $\rho=\rho_{0} R_{0}^{2} / R^{2}=\dot{M} / 4 \pi R^{2} V$ where the typical mass-loss rate $\dot{M} \sim 10^{-4} M_{\odot} \mathrm{y}^{-1}$ and the typical wind velocity $V \sim 1000 \mathrm{~km} \mathrm{~s}^{-1}$ yield $\rho_{0} R_{0}^{2}=5 \times 10^{11} \mathrm{~g} \mathrm{~cm}^{-1}$. The replacement of $n_{\mathrm{b}} m_{\mathrm{p}} R^{2}$ with $\rho_{0} R_{0}^{2}$ in the Eq. (A.1), and repetition of the derivations of the break-time correlations for a constant density, yield, for $\gamma \theta=1$,

$$
R_{\mathrm{b}} \approx \frac{E_{\mathrm{k}}}{2 \pi \rho_{0} R_{0}^{2} c^{3}}
$$

i.e., a typical $R_{\mathrm{b}} \approx 3.5 \times 10^{16}$ independent of $\theta$, and

$t_{\mathrm{b}}^{\prime} \approx \frac{\theta^{2} E_{\mathrm{k}}}{8 \pi \rho_{0} R_{0}^{2} c^{3}}=\frac{\eta E_{\mathrm{k}}^{2}}{2 \pi \rho_{0} R_{0}^{2} c^{3}} \frac{1}{E_{\mathrm{iso}}}$.

The break-time power law correlations for ISM and wind-like density profiles thus have identical power law indices, i.e., $t_{\mathrm{b}}^{\prime} \propto$ $1 / E_{\text {iso }} \propto 1 / E_{\mathrm{p}}^{\prime}$.

\section{Appendix B: Ballistic cannonballs}

In the $\mathrm{CB}$ model the electrons that enter the $\mathrm{CB}$ are Fermiaccelerated, and they cool rapidly by synchrotron radiation (SR). This radiation is isotropic in the CB's rest frame and has a smoothly broken power law spectrum with a characteristic bend/break frequency, which is the typical synchrotron frequency radiated by the ISM electrons that enter the CB at time $t$ with a relative Lorentz factor $\gamma(t)$. In the observer frame, the emitted photons are beamed into a narrow cone along the CB's direction of motion by its highly relativistic bulk motion, their arrival times are aberrated and their energies are boosted by its bulk motion Doppler factor $\delta$ and redshifted by the cosmic expansion during their travel time to the observer. For the X-ray band that is well above the break frequency, the CB model yields the spectral energy density (see, e.g., Eq. (26) in Dado et al. 2009a),

$$
F_{v} \propto n^{\left(\beta_{\mathrm{X}}+1\right) / 2}[\gamma(t)]^{3 \beta_{\mathrm{X}}-1}[\delta(t)]^{\beta_{\mathrm{X}}+3} v^{-\beta_{\mathrm{X}}} .
$$

For a $\mathrm{CB}$ of a baryon number $N_{B}$, a constant or slowly expanding radius $R$ and an initial Lorentz factor $\gamma_{0}=\gamma(0) \gg 1$, which propagates in an ISM of a constant density $n_{\mathrm{b}}$, relativistic energymomentum conservation yields the deceleration law (Dado et al. 2009b, and references therein)

$\gamma(t)=\frac{\gamma_{0}}{\left[\sqrt{\left(1+\theta^{2} \gamma_{0}^{2}\right)^{2}+t / t_{0}}-\theta^{2} \gamma_{0}^{2}\right]^{1 / 2}}$,

where $t_{0}=(1+z) N_{\mathrm{B}} / 8 c n \pi R^{2} \gamma_{0}^{3}$. As long as $t<t_{\mathrm{b}}=\left(1+\gamma_{0}^{2} \theta^{2}\right)^{2} t_{0}$, $\gamma(t)$ and $\delta(t)$ change rather slowly with $t$, which generates the plateau phase of $F_{v}(t)$ of canonical X-ray AGs that was predicted by the CB model (see, e.g., Dado et al. 2002, Figs. 6, 27-33) and later observed with Swift (Nousek et al. 2005). For $t \gg t_{\mathrm{b}}$, $\gamma(t) \rightarrow \gamma_{0}\left(t / t_{\mathrm{b}}\right)^{-1 / 4},[\gamma(t) \theta]^{2}$ becomes $\ll 1$ and $\delta \approx 2 \gamma(t)$, which result in a post-break power law decline

$F_{v}(t) \propto v^{-\beta_{\mathrm{X}}} t^{-\left(\beta_{\mathrm{X}}+1 / 2\right)}$. 
Thus, in the CB model, the asymptotic post-break decline of the $\mathrm{X}$-ray afterglow of a single $\mathrm{CB}$ in an ISM environment satisfies the closure relation $\alpha_{\mathrm{X}}=\beta_{\mathrm{X}}+1 / 2=\Gamma_{\mathrm{X}}-1 / 2$ (or $\alpha_{\mathrm{X}}=\beta_{\mathrm{X}}=$ $\Gamma_{\mathrm{X}}-1$ for a shot-gun configuration of CBs) independent of the pre-break behavior, which strongly depends on viewing angle (e.g., Dado \& Dar 2012a).

\section{References}

Amati, L., Della Valle, M., Frontera, F., et al. 2007, A\&A, 463, 913A Amati, L., Guidorzi, C., Frontera, F., et al. 2008, MNRAS, 391, 577 Barthelmy, S. 1997, http://gcn.gsfc.nasa.gov/gcn_main.html Beuermann, Hessman, F. V., Reinsch, K., et al. 1999, A\&A, 352, L26 Costa, E., Frontera, F., Heise, J., et al. 1997, Nature, 387, 783

Covino, S., Malesani, D., Tagliaferri, G., et al. 2006, IL Nuovo Cimento B, 121, 1171

Curran, P. A., van der Horst, A. J., \& Wijers, R. A. M. J. 2008, MNRAS, 386, 859

Dado, S., \& Dar, A. 2012a, ApJ, 761, 148

Dado, S., \& Dar, A. 2012b, ApJ, in press [arXiv: 1203.5886]

Dado, S., Dar, A., \& De Rújula, A. 2002, A\&A, 388, 1079

Dado, S., Dar, A., \& De Rújula, A. 2004, A\&A, 422, 2004

Dado, S., Dar, A., \& De Rújula, A. 2007, ApJ, 663, 400

Dado, S., Dar, A., \& De Rújula, A. 2009a, ApJ, 696, 994

Dado, S., Dar A., \& De Rújula A. 2009b, ApJ, 693, 311

D’Agostini, G. 2005 [arXiv: astro-ph/0511182]

Dar, A. 1998, ApJ, 500, L93

Dar, A., \& De Rújula A. 2000 [arXiv: astro-ph/0008474]

Dar, A., \& De Rújula, A. 2004, Phys. Rep. 405, 203

D’Avanzo, P., Salvaterra, R., Sbarufatti, B., et al. 2012, MNRAS, 425, 506

Evans, P. A., Beardmore, A. P., Page, K. L., et al. 2009, MNRAS, 397, 1177

Frail, D. A., Kulkarni, S. R., Nicastro, L., et al. 1997, Nature, 389, 261
Frail, D. A., Kulkarni, S. R., Sari, R., et al. 2001, ApJ, 562, L55

Fruchter, A. S., Thorsett, S. E., Metzger, M. R. A., et al. 1999, ApJ, 519, L13

Goodman, J., 1986, ApJ, 308, L47

Goodman, J., Dar, A., \& Nussinov, S. 1987, ApJ, 314, L7

Gruber, D., Greiner, J., von Kienlin, A., et al. 2011, A\&A, 531A, 20

Harrison, F. A., Bloom, J. S., Frail, D. A., et al. 1999, ApJ, 523, L121

Katz, J. 1994, ApJ, 432, L107

Kocevski, D., \& Butler, N. 2008, ApJ, 680, 531

Kouveliotou, C., Woosley, S. E., Patel, S. K., et al. 2004, ApJ, 608, 872

Kulkarni, S. R., Djorgovski, S. G., Odewahn, S. C., et al. 1999, Nature, 398, 389 Liang, E. W., Racusin, J.L., Zhang, B., et al. 2008, ApJ, 675, L528

Margutti, R., Zaninoni, E., Bernardini, M. G., et al. 2013, MNRAS, 428, 729

Meegan, Fishman, G. J., Wilson, R. B., et al. 1992, Nature, 355, 143

Meszaros, P., \& Rees, M. J. 1997, ApJ, 476, 232

Metzger, M. R., Djorgovski, S. G., Kulkarni, S. R., et al. 1997, Nature, 387, 878

Mao, S., \& Paczynski, B. 1992, ApJ, 388, L45

Nava, L., Salvaterra, R., Ghirlanda, G., et al. 2012, MNRAS, 421, 1256

Nousek, J. A., Kouveliotou, C., Grupe, D., et al. 2006, ApJ, 642, 389

Paczynski, B. 1986, ApJ, 308, L43

Paczynski, B., \& Rhoads, J. E. 1993, ApJ, 418, L5

Panaitescu, A., Mészáros, P., Burrows, D., et al. 2006, MNRAS, 369, 2059

Piran, T. 1999, Phys. Rep. 314, 575

Piran, T. 2000, Phys. Rep. 333, 529

Racusin, J. L., Liang, E. W., Burrows, D. N., et al. 2009, ApJ, 698, 43

Sahu, K. C., Livio, M., Petro, L., et al. 1997, Nature, 387, 476

Sari, R., Piran, T., \& Halpern, J. P. 1999, ApJ, 519, L17

Shaviv, N. J., \& Dar, A. 1995, ApJ, 447, 863

Stratta, G., Guetta, D. , D'Elia, V., et al. 2009, A\&A, 494, L9

Van Eerten, H. J., MacFadyen, A. I., \& Zhang, W. 2011, AIP Conf. Proc., 1358, 173

van Eerten, F., \& MacFadyen, A. 2013, ApJ, 767, 141

van Paradijs, J., Groot, P. J., Galama, T., et al. 1997, Nature, 386, 686

Wijers, R. A. M. J., Rees, J., \& Meszaros, P. 1997, MNRAS, 288, L51

Yonetoku, D., Murakami, T., Tsutsui, R., et al. 2010, PASJ, 62, 1495 